\title{
Rbx1 Cullin-Ring ligase E3 and Ube2m neddylation E2 differentially governs the fitness of Treg cells
}

Di Wu${ }^{1}$, Haomin $\mathrm{Li}^{2}$, Mingwei $\mathrm{Liu}^{3}$, Jun Qin ${ }^{3}$, Yi Sun ${ }^{1 *}$

${ }^{1}$ Cancer Institute of the Second Affiliated Hospital and Institute of Translational Medicine, Zhejiang University School of Medicine, Hangzhou, Zhejiang, China, 310029

${ }^{2}$ Children's Hospital, Zhejiang University School of Medicine, Hangzhou, Zhejiang, China, 310003

${ }^{3}$ State Key Laboratory of Proteomics, Beijing Proteome Research Center, National Center for Protein Sciences (Beijing) and Institute of Lifeomics, Beijing, China, 102206

* To whom correspondence should be addressed: yisun@zju.edu.cn 
Cullin-RING Ligases (CRLs) are a family of multi-unit E3 ubiquitin ligases with two members of RING family proteins, acting as the catalytic subunit: RING-box 1 (Rbx1) couples with CRLs1-4, whereas RING-box 2 (Rbx2/Sag) couples mainly with CRL5. ${ }^{1-4}$ Activity of CRLs requires neddylation on their Cullin subunit, catalyzed by neddylation enzyme cascades E1, E2 and E3. Ube2m and Ube2f are two neddylation E2s responsible for neddylation of Cullins in CRLs1-4 or CRL5, respectively. ${ }^{5-6}$ Regulatory $T$ cells (Treg cells) are specialized immunosuppressive $\mathrm{CD}^{+} \mathrm{T}$ lymphocytes, that play pivotal roles in maintaining immune homeostasis in vivo. ${ }^{7-10}$ Whether and how Rbx1-Rbx2/CRLs and Ube2m-Ube2f/neddylation regulate Treg cell homeostasis and function are currently unknown. Here we show that while mice with a Treg-specific deletion of $R b x$ 2/Sag showed no obvious phenotype, mice with $R b x 1$ deletion in Treg cells developed an early-onset fetal inflammatory disorders and death at day $\sim 25$ after birth ( p 25), with disrupted homeostasis and functions of Treg cells, indicating Rbx1 as a prominent regulator of Treg cells. Single cell transcriptome assay showed that $R b x 1$ is essential for the maintenance of the effector subpopulations in Treg cells. The whole genome transcriptome and proteomics analysis revealed that $\mathrm{Rbx1}$ regulates several inflammatory pathways, such as T-cell receptor, IL-17, TNF $\alpha$, NFкB, chemokine, cytokine-cytokine receptor interaction, as well as energy and purine metabolisms. Accumulation of Acly, Fto and Nfkbib proteins, upon Rbx1 depletion suggests that these are likely the novel substrates of CRLs1-4 in Treg cells. Consistently, while Ube $2 f$ deletion showed no obvious phenotype, mice with Ube2m deletion in Treg cells also suffers from inflammatory disorders, but to a much lesser severity with a $50 \%$ of death rate at 150 days of age. Since $R b x 1$ is a dual $E 3$ as a component of CRLs1-4 ligase and as a neddylation co-E3, downstream of Ube2m E2 for neddylation activation of CRLs1-4, much severe phenotypes in $F_{0 x p 3^{c r e}}$; $B b x 1^{f l f l}$ mice suggests $R b x 1$ may have additional function independent of neddylation activation in Treg cells. Indeed, unbiased transcriptome comparison between $R b x 1$-deficient and Ube2m-deficient Treg cells, revealed that the former had greater as well as unique alteration in the signaling pathways controlling the inflammatory responses. Collectively, our study shows that the Ube2m-Rbx1 axis of the neddylation-CRL is required for the maintenance of homeostasis and functional fitness of Treg cells in the fine control of immune tolerance; with implication that targeting the neddylation/CRLs, such as a small molecule inhibitor pevonedistat $^{11}$, currently in the Phase II clinic trial for anticancer therapy, may have novel application in the treatment of human diseases associated with overactivated Treg cells. 
A Cullin-RING ligase (CRL) ubiquitin E3 complex consists of 4 subunits: a scaffold Cullin with 8 family members, an adaptor with many members, a substrate receptor with hundreds of members, and a RING catalytic component with two family members, RING-box 1 (Rbx1/Roc1) and RING-box 2 (Rbx2/Roc2/Sag). ${ }^{12}$ Our previous total knockout study showed that $R b x 1$ and $R b \times 2 / S a g$ are functionally non-redundant and required for mouse embryogenesis ${ }^{13-14}$. Although mice are viable, T-cell specific Sag knockout (via Lck-Cre) significantly decreased T cell activation, proliferation, and T-effector cytokine release ${ }^{15}$, whereas Sag knockout in myeloid lineage (via LysM-Cre) increased serum levels of proinflammatory cytokines and enhanced mortality in response to LPS ${ }^{16}$.

To investigate the role of CRLs in regulation of Treg cells in vivo, we generated conditional knockout mouse models with inactivation of $R b x l$ and Rbx2/Sag individually in Treg cells, driven by Foxp3 $3^{\text {YFP-cre }}\left(F_{\text {oxp }} 3^{\text {cre }}\right)^{17,18}$. The compound mice were designated as $F_{o x p} 3^{\text {cre }} ; R b x l^{f l f l}$ or $F_{o x p} 3^{\text {cre }} ;$ Sag ${ }^{f l / f l}$ mice, respectively. Compared with Foxp3 $3^{\text {cre }}$ controls (wild-type/wt), Foxp3 ${ }^{\text {cre }} ;$ Sag ${ }^{\text {fllfl }}$ mice were viable and healthy without obvious alterations in activation markers of $\mathrm{CD}^{+}{ }^{+}$Foxp $^{-}{ }^{-} \mathrm{T}$ cells (conventional $\mathrm{T}$ cells, or Tcon cells), Tcon cell proliferation rate, and the Treg $/ \mathrm{CD}^{+}$ratio (Extended Data Fig. 1), indicating that Treg specific depletion of Sag does not obviously impair Treg cell function and survival at the steady state.

\section{Early-onset fatal inflammation in $F_{o x p} 3^{\text {cre }} ;\left.R b x\right|^{f l f l}$ mice}

Strikingly, deletion of $R b x l$ (confirmed by transcriptome profiling in $\mathrm{CD}^{+} \mathrm{YFP}^{+}$Treg cells with 100-fold reduction of Rbxl mRNA, Extended data Fig. 2a) in Treg cells was early-onset fatal. Compared with wt controls, Foxp $3^{\text {cre }} ; R b x 1^{f l f l}$ mice showed an altered appearance as early as day 13-15 after birth (p13-15), with collapsed ears, festered skin, and reduced body size (Fig.1a). The mice continued to lose weight dramatically after p15 (Fig. 1 b) with a $50 \%$ or $100 \%$ of death rate at p25 or p37, respectively (Fig.1c). Autopsy examination revealed that Foxp $3^{\text {cre }} ; R b x 1^{\text {flffl }}$ mice had swollen lymph nodes and spleens (Fig. 1d \& Extended data Fig. 2b), with lymphocyte infiltration into multiple organs, including the skin, lung, stomach, liver, kidney and colon (Extended data Fig. 2c). The Foxp $3^{\text {cre }} ; R b x 1^{f l f l}$ mice also had a decreased $\mathrm{CD}^{+} / \mathrm{CD}^{+}$T-cell ratio (Extended data Fig. 2d) and a significantly increased proportion of effector/memory $\mathrm{T}$ cells $\left(\mathrm{CD} 44{ }^{\mathrm{hi}} \mathrm{CD} 62 \mathrm{~L}^{\mathrm{lo}}\right)$ among Tcon cells (Fig.1e \& Extended data Fig.2e), indicating robust activation of immune cells, a typical phenotype of autoimmune disease. The fatal inflammation observed in Foxp $3^{\text {cre }}$;Rbx $1^{\text {flfl }}$ mice is reminiscent of Treg-deficient mice $^{10}$ or mice with loss-of-function mutations in the Foxp 3 gene $^{19}$, suggesting Rbxl is absolutely essential for Treg cells in vivo. 


\section{Rbx1 maintains Treg cell homeostasis and suppressive function}

In moribund Foxp $3^{\text {cre }} ;$ Rbx $I^{\text {flffl }}$ mice (p19-23), the Treg cell compartment was reduced (Fig.1f \& Extended data Fig.3a), with reduced Ki67 expression (Fig.1g\& Extended data Fig.3b) and increased caspase-3 activity (Fig.1h\& Extended data Fig.3c), indicating reduced proliferation and increased apoptosis. The levels of Ifn- $\gamma$ and Il-4 were remarkably increased in Tcon cells by flow cytometry detection of single cell suspension from lymph-nodes (Fig.1i). In addition, the serum concentrations of $\mathrm{T}_{\mathrm{H}} 1 / \mathrm{T}_{\mathrm{H}} 2$ cytokines (Ifn- $\gamma$, Il-2 for $\mathrm{T}_{\mathrm{H}} 1$; Il-4, Il-5, Il6, Il-9, Il-10, and Il-13 for $\mathrm{T}_{\mathrm{H}} 2$ ) and antibodies ( $\operatorname{IgA}$ and $\operatorname{IgG} 2 \mathrm{a}$ for $\mathrm{T}_{\mathrm{H}} 1$; IgE and $\operatorname{IgG} 1$ for $\mathrm{T}_{\mathrm{H}} 2$ ) were elevated (Extended data Fig.4a\&b and Extended data Fig.5a\&b), indicating a robust activation of $\mathrm{T}_{\mathrm{H}} 1 / \mathrm{T}_{\mathrm{H}} 2$-immune responses. For $\mathrm{T}_{\mathrm{H}} 17$ reaction, flow cytometry of single cell suspension from lymph-nodes revealed a 2-fold, but statistically significant increase of Il-17A in Tcon cells, (Fig.1i) and serum concentrations of $\mathrm{T}_{\mathrm{H}} 17$ antibodies (IgG3, IgG2b) were also elevated, although not statistically different (Extended data Fig. 5c). Although the serum concentrations of $\mathrm{T}_{\mathrm{H}} 17$ cytokines (Tnf- $\alpha$, and GM-Csf) did not change obviously, Il-17A level was reduced, whereas the robust inflammatory storm was evident with significantly increased amount of various cytokines in Foxp3 ${ }^{\text {cre }} ;$ Rbx $l^{f l f l}$ mice (Extended data Fig. 4c\&d). Thus, while the $\mathrm{T}_{\mathrm{H}} 17$ reaction was activated modestly, an excessive $\mathrm{T}_{\mathrm{H}} 1 / \mathrm{T}_{\mathrm{H}} 2$-dominant immune polarization was observed in Foxp $3^{\text {cre }} ;$ Rbx $1^{\text {flffl }}$ mice.

To determine early alterations, we used the $F_{\text {oxp }} 3^{\text {cre }} ; R b x 1^{f l f l}$ mice at $\mathrm{p} 8$ stage without apparent inflammatory disorders. While these mice had largely normal proportion of Treg cells among $\mathrm{CD}^{+}-\mathrm{T}$ cells, the overactivation of T-cells were readily detectable, as evidenced by an increased proportion of effector/memory $\left(\mathrm{CD} 44^{\mathrm{hi}} \mathrm{CD} 62 \mathrm{~L}^{\mathrm{lo}}\right) \mathrm{T}$ cells and increased Ki67 levels in Tcon cells (Fig. 1j-1). Thus, $\mathrm{Rbx} 1$ is indeed required for the maintenance of suppressive function of Treg cell population.

\section{Reduction of effector subpopulation of Treg cells upon $R b x \boldsymbol{l}$ deletion}

Single-cell RNA sequencing (scRNA-seq) was utilized to unbiasedly dissect the cellular heterogeneity of $w t$ and $R b x l$-deficient $\mathrm{CD}^{+} \mathrm{YFP}^{+}$Treg cells from the peripheral lymph nodes of inflammation-free Foxp $3^{\text {cre/wt }}$ and Foxp $3^{\text {cre/wt }}$;Rbx $1^{\text {fllfl }}$ mice (Extended data Fig. 6). As revealed in the two-dimensional Uniform Manifold Approximation and Projection (UMAP) of the single-cell transcriptomics data, both $w t$ and Rbxl-deficient Treg cells could be divided into several subpopulations (Fig.2a \& Extended data Fig. 7). Rbx1-depletion increased the subpopulation in Cluster 0, whereas remarkably decreased the subpopulations on Clusters 3 and 4 without affecting other subpopulations obviously (Fig.2b).

The cells in Cluster 0 expressed high levels of genes characteristic of naive Treg cells, including Sell ${ }^{20}$ and Bcl2; while Treg cell functional molecules, such as 
$\mathrm{Ctla}^{21}{ }^{2}, \operatorname{Icos}^{22}$, and $C d 44^{23}$, were highly expressed in Cluster 3 and 4 (Fig.2c\& Extended data Fig. 8). Thus, Cluster 0 is a quiescent subpopulation and designated as the center population ${ }^{24}$, whereas Clusters 3 and 4 are active Treg cell subpopulations designated as effector populations. The KEGG pathway enrichment analysis revealed that, compared to Cluster 0, T-cell receptor ${ }^{25}$ and other Treg related pathways were activated in Cluster 3 and 4, (Fig. 2d\& Extended data Fig. 9). Taken together, Rbx1 is, therefore, essential for the maintenance of effector subpopulations of Treg cells. Lack of effect of Rbx1-deficiency on other subpopulations in Treg cells provides an ideal opportunity of Rbx1 targeting for precise manipulation of Treg cell subpopulations.

\section{Disordered functional and regulatory network in Rbx1-deficent Treg cells}

Transcriptome analysis of Rbx1-deficient Treg cells from inflammation-free Foxp3 ${ }^{\text {cre/wt }}$;Rbx $1^{f l f l}$ mice (Extended data Fig. 6) revealed significantly reduced expression of functional genes (Fig. 3a), including $1 l 10^{17}, T g f b^{26}$, and $\operatorname{Cst}^{27}$ (suppressive cytokines); $I l 2 r^{28}$ and Entpd $1^{29-30}$ (regulators of immune cell metabolism); $\mathrm{Ctla}^{22}$, Icos ${ }^{23}, \mathrm{Nrpl}^{31}$, and $\mathrm{Lag}^{32}$ (inhibitor via dendritic cells); and $\mathrm{Fas}^{33}$ and Lgals $1^{34}$ (apoptosis inducer). Impairment of migration-associated surface molecules was also observed in $R b x l$-deficient Treg cells, including down-regulation of $C d 44$, Cxcr3, Cxcr5, and Cxcr6 ${ }^{23}$ and up-regulation of Sell ${ }^{21}$.

The KEGG pathway enrichment analysis revealed that Rbx1 depletion caused downregulation of transcriptome involved in many signaling pathways, including T-cell receptor, Th17 cell differentiation, chemokine, cytokine-cytokine receptor interaction, NOD-like receptor, TNF, FoxO, Toll-like receptor, Hippo, TGF $\beta$, NF- $\kappa B$, PI3K-AKT, and purine metabolism among few other virus associated pathways (Fig. $3 \mathrm{~b} \&$ Extended data Fig. 10). The results strongly suggest Rbx1 regulation of numerous genes controlling the inflammation, immunological responsiveness, proliferation/survival, and metabolism.

\section{Specific spectrum of Rbx1 substrates in Treg cells}

Considering Rbx1 is an E3 ubiquitin ligase that directly controls the abundance of substrate proteins, we utilized the mass spectrometry-based proteomics to profile the difference in protein amounts between $\mathrm{Wt}$ and $\mathrm{Rbx} 1$-deficient $\mathrm{CD}^{+} \mathrm{YFP}^{+}$Treg cells (three independent sets of combined samples) from inflammation-free $F_{o x p} 3^{\text {crelwt }}$ and $F_{o x p} 3^{\text {cre/wt }}$;Rbx $1^{\text {flflfl }}$ mice (Extended data Fig. 6). Among a total of 3236 proteins detected in any one of 6 paired samples, we identified 43 increased and 78 decreased proteins with a 3-fold cut-off, and the numbers increased to 70 and 195, respectively, with a 2-fold cut-off in Rbx1-deficient $\mathrm{CD}^{+} \mathrm{YFP}^{+}$Treg cells, as compared to the wild-type. The KEGG pathway enrichment analysis of 2-fold cut-off revealed that Rbx1 depletion caused accumulation of few proteins associated with the splicesome or complement and coagulation cascade 
(Extended data Fig. 11), but significant reduction of proteins controlling TCA cycle, carbon and purine metabolisms, along with other metabolic pathways (Fig. 3c), suggesting a requirement of Rbx1 in regulation of energy and nucleotide metabolisms of Treg cells. We noticed that less than half of protein changes seen in the proteomic analysis matched with the mRNA changes by the transcriptomic analysis (Fig. 3c \& Extended data Fig. 11), which is slightly lower than the general correlation of $50 \%$ between proteome and transcripteome ${ }^{35}$.

To evaluate the direct substrates of Rbx1 ubiquitin E3 ligase in Treg cells, the proteomic and transcriptomic data were combined for analysis. The candidates are those with increased levels of protein but not the mRNA in Rbx1-deficient Treg cells, which include Acly, Manf, Mlec, As3mt, Fto, and Nfkbib, among the others (Fig. 3d). Acly ${ }^{36}$ and Nfkbib/IkB- $\beta^{37}$ were previously reported as Rbx1 substrates, whereas the others are likely the novel substrates. Interestingly, many common substrates of Rbx1/CRLs 1-4 ${ }^{38-39}$ were not detected in Rbx1 deficient Treg cells, few detected ones, like Cdkn1b/p27 $7^{13}$ and Foxo1 ${ }^{40}$, were not accumulated (Extended data Fig. 12), indicating that a subset of Rbx1 substrates are selectively expressed, and specifically subjected to Rbx1 modulation in Treg cells.

Among the putative substrates of Rbx1-CRLs1-4 functioning uniquely in Treg

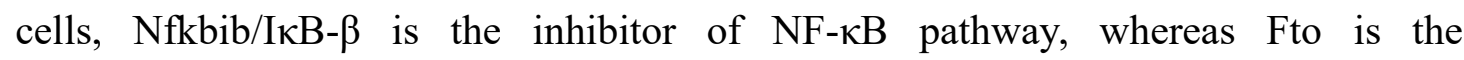
demethylation enzyme of $\mathrm{m}^{6} \mathrm{~A}$ mRNA methylation. NF- $\kappa \mathrm{B}$ pathway ${ }^{18,41}$ and $\mathrm{m}^{6} \mathrm{~A}$ mRNA methylation ${ }^{42}$ are known to regulate Treg cell functions. Their accumulation upon Rbx 1 depletion is likely contributing to observed malfunctions of Treg cells.

\section{Rbx1 acts in Treg cells via Ube2m-dependent and-independent mechanisms}

It has been well-established that the E3 ligase activity of the CRLs requires neddylation of the scaffold protein Cullin ${ }^{6}$. Specifically, neddylation E2 Ube $2 \mathrm{~m}$ couples with Rbx1 (neddylation co-E3) selectively promotes neddylation on Cullins1-4, which are associated with Rbx1 to constitute active CRLs1-4 ubiquitin E3; whereas neddylation E2 Ube2f couples with $\mathrm{Sag} / \mathrm{Rbx} 2 \mathrm{E} 3$ to promote neddylation of Cullin5, which is associated with Rbx2 to constitute active CRL55. We, therefore, asked whether the pivotal role of Rbx1 in Treg cells is dependent on Ube2m, whereas Ube2f is non-essential for Treg cells, given the non-essentiality of Sag/Rbx2. Indeed, Foxp $3^{\text {cre }}$,Ube $2 f^{\text {fllfl }}$ mice are viable and develop normally without any abnormality (Extended data Fig. 13), indicating that the Ube2f-Rbx2-CRL5 axis is not essential for the functionality and survival of Treg cells at the steady status.

Consistently, like Foxp $3^{\text {cre }} ; R b x 1^{f l f l}$ mice, Foxp $3^{\text {cre }} ; U b e 2 m^{\text {fllfl }}$ mice with Ube $2 m$ deletion in Treg cells (again confirmed by transcriptome profiling, Extended data Fig. 14a), displayed abnormal appearances such as collapsed ears and festered skin, but at the much later stage (Fig. 4a). The growth of mice was remarkably retarded (Fig. 4b), and about $50 \%$ of the mice dies at age of 150 days and a $100 \%$ death rate within one 
year (Fig. 4c). The swollen spleens and lymph nodes were apparent (Fig. 4d \& Extended data Fig.14b). Foxp3 $3^{\text {cre }}$,Ube $2 m^{\text {flffl }}$ mice also showed obvious $\mathrm{T}_{\mathrm{H}} 1 / \mathrm{T}_{\mathrm{H}}$ 2-polarized immune over-activation but in a much lower levels of inflammation than that in Foxp $3^{\text {cre }} ; R b x l^{f l f l}$ mice (Fig.4e\&f, Extended data Fig.14-16), but had increased Treg/CD4 $4^{+} \mathrm{T}$ ratio, and lack of Ki67-decrease and active-Casp3-increase in Treg cells (Extended data Fig.17). These observations demonstrate the similarities and differences between the functions of Rbx1 and Ube $2 \mathrm{~m}$ in Treg cells.

The genome-wide transcriptome analyses of Ube2m-deficient Treg cells, as compared to Rbxl-deficient Treg cells, revealed the similar changes in the Treg functional genes (Extended data Fig.18), controlling multiple signaling pathways involved in inflammation responses (Extended data Fig.19), but generally to a lesser extent. An unbiased cluster analysis of the transcriptomes between $R b x l$-deficient and Ube $2 m$-deficient Treg cells revealed a good overall correction, but two sets of data did have unique changes in several groups of genes specifically seen in $R b x l$-deficient Treg cells (Fig.4g and Extended data Fig.20\&21), suggesting the dependent and independent mechanisms between $\mathrm{Rbx} 1$ and Ube $2 \mathrm{~m}$ in regulation of Treg cell functions. Taken together, both the phenotype comparison and unbiased cluster analyses of the transcriptome support the notion that Rbx1 acts via Ube2m-dependent and -independent mechanisms in Treg cells.

\section{Discussion}

Many preclinical studies have demonstrated that overactivation of protein neddylation and CRL E3s are common in human cancers, which is actively involved in promoting tumorigenesis. ${ }^{12}$ Pevonedistat (also known as MLN4924), a small molecule inhibitor of neddylation E1, that blocks the entire protein neddylation and inhibites all CRL E3s, is currently in several phase II clinical trials for anti-cancer application. ${ }^{39}$ We showed here that inactivation of neddylation by depletion of Ube $2 \mathrm{~m}$ E2 or inactivation of CRLs1-4 by depletion of Rbx1 in Treg cells dramatically inhibits functions and survival of Treg cells, leading to development of the autoimmune disorders with overactivated $\mathrm{T}$ toxic cells and premature death. The Treg Rbx1-deficient mice have much severer phenotype than the Ube2m-deficient mice suggest Rbx1/CRLs1-4 may have additional function beyond neddylation activation. Lack of phenotype by $R b x 2 / S a g$ or $U b e 2 f$ deletion in Treg cells excludes possible involvement of CRL5 in Treg regulation.

While both Rbx1 and Ube2m controls the expression of genes regulating several inflammation-related pathways (Extended data Fig.19), the downregulation of $\operatorname{Egr}^{43}, \mathrm{Ccr}^{44}$ and Tigit ${ }^{45}$ appear to be relatively unique to Rbx1-deficient Treg cells (Extended data Fig. 20). Altered expression of these genes, which has been shown to regulate Treg cell function, would likely be responsible for the more severe 
phenotypes seen in $F o x p 3^{\text {cre }} ; R b x 1^{f l f l}$ mice. The KEGG pathway analysis of a group of genes uniquely responded to Rbxl-deficiency revealed Rbxl regulation of several biosynthesis processes and cell-cell adherent junctions, among few others, (Extended data Fig.21) which is an interesting subject for future investigation. More important, Rbx1 was found to control several metabolic pathways, particularly purine pathways shown in both proteomics and transcriptomics analyses (Fig. 3c), suggesting reduced operation of these pathways upon Rbx1 depletion may also contribute to the loss of Treg function and eventually death of animals.

In summary, our study revealed that Rbx1 is essential for the functionality and survival of Treg cells by controlling multiple inflammatory and proliferation/survival signaling, as well as metabolic pathways. Our study also provided a piece of proof-of-concept evidence that the neddylation inhibitor pevonedistat or selective targeting Treg-RBX1, may have novel application in the treatment of human diseases associated with overactivation of Treg cells by inactivating of neddylation/CRLs.

\section{Acknowledgments}

We would like to thank Prof. Xiaoming Feng for providing Foxp $3^{\text {YFP-cre }}$ mice. We also thank the Core Facilities, Zhejiang University School of Medicine for the technical support. This project was supported by the National Key R\&D Program of China (2016YFA0501800 to Y. S.); National Science Foundation of China (81801567 to D. W. and 81572718 to Y. S.).

\section{Author contributions}

Di Wu designed and preformed experiments, analyzed data, and wrote the manuscript. Haomin Li analyzed proteome and transcriptome data. Mingwei Liu performed the mass spectrum experiment and analyzed the data. Jun Qin designed and supervised the mass spectrum experiment and analyzed data. Yi Sun designed experiments, analyzed data, wrote the manuscript, and oversaw the project.

\section{Competing interests}

The authors declare no competing interests. 


\section{METHODS}

\section{Data reporting}

No statistical methods were used to predetermine sample size. The experiments were not randomized and the investigators were not blinded to allocation during experiments and outcome assessment.

\section{Mice}

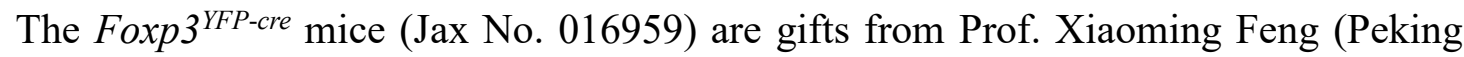
Union Medical College, China).

The $R b x l^{f l f l}$ mice were generated on the C57BL/6 background via CRISPR/Cas9 technology with exons 2-4 floxed (performed by Nanjing Biomedical Research Institute of Nanjing University). Briefly, the sgRNA (5'-A CCACTAAGTGGATAAT CAC-3' and 5'-GGAGTCAGAAATGATTGGTC-3') direct Cas9 endonuclease cleavage in intron1-2 and intron 4-5 to insert LoxP sites by homologous recombination. The PCR genotyping primers 5'-GATTCTACTTTGCTTGCAGTGC TC-3' and 5'-TCTGCATAAGCACGGGCTCTC-3' generated DNA fragment of 266bp and 196bp bands, respectively, for mutant and wild type.

The Ube $2 m^{f l f l}$ mice were generated on the C57BL/6 background via CRISPR/Cas9 technology with exons 2-4 floxed (performed by Nanjing Biomedical Research Institute of Nanjing University). Briefly, the sgRNA (5'-TCAATTTAAGCTACCATA C-3' and 5'-AGTGAAGATACGGGACA-3') direct Cas9 endonuclease cleavage in intron 1-2 and intron 4-5 to insert LoxP sites by homologous recombination. The PCR genotyping primers 5'-CAAGACCTGCCTTCCAGGTATC-3' and 5'-CCCTGCTAA TACTGAACAGG-3' generated DNA fragment of 317bp and 223bp for mutant and wildtype, respectively,

The Ube2flfll mice were generated from an ES clone (HEPD0820_4_G09), purchased from EuMMCR (European Mouse Mutant Cell Repository) (MGI:1915171) with exon 5 floxed. For LoxP sites flanked allele, the PCR genotyping primers are 5'-GCGAGCTCAGACCATAACTTCG-3' and 5'-CCAGGGTGGAAAATTTCAGT TT-3'; and for wild type allele, the genotyping primers are 5'-CCCTGGAATTTCGG TATTATA-3' and 5'-CCAGGGTGGAAAATTTCAGTTT-3', respectively.

The $S a g^{f l f l}$ mice were generated and characterized as described ${ }^{46}$.

All mice were maintained in SPF conditions. All animal experiments were approved by the Animal Ethics Committee of Zhejiang University; animal care was provided in accordance with the principles and procedures by the regulatory standards at Zhejiang University Laboratory Animal Center. 


\section{Flow cytometry}

For analysis of surface markers, cells were washed in PBS containing 2\% (wt/vol) FBS, and stained with indicated Abs according to the manufacturer's instructions (BD Pharmingen, 562574), followed by flow cytometry analysis. For intracellular cytokine staining, cells were stimulated for $5 \mathrm{~h}$ with leukocyte activation cocktail of GolgiPlug (BD Pharmingen, 550583). Antibodies were all from eBioscience, unless otherwise noted: anti-CD4 (GK1.5), anti-CD8 $\alpha$ (53-6.7, BD Pharmingen), anti-CD44 (IM7, BD Pharmingen), anti-CD62L (MEL-14), anti-Foxp3 (FJK-16s), anti-Ki67 (SolA15), anti-active caspase 3 (C92-605, BD Pharmingen), anti-Ifn- $\gamma$ (XMG1.2), anti-Il4 (11B11), anti-I117A (eBio17B7). Flow cytometry data were acquired on CytoFLEX LX or CytoFLEX S (Beckman) and analyzed using Flowjo software (Tree Star).

\section{Cytokines assay}

The serum was drawn from mice of indicated genotypes, diluted 4-fold by PBS, and then subjected to cytokine/chemokine profiling using Mouse Cytokine Grp I (Bio-Plex Pro, M60009RDPD), measured on Luminex 200 system.

\section{Antibodies assay}

The immunoglobulin subclasses in mouse serum were measured using Mouse Immunoglobulin Isotyping Kit (BD Cytometric Bead Array, 550026).

\section{Cell sorting}

Peripheral lymph nodes and/or spleens were harvested from mice with indicated genotypes, and grinded into single cells, followed by treatment with Dynabeads Untouched Mouse CD4 Cells Kit (Invitrogen, 11415D) to isolate the CD4 ${ }^{+} \mathrm{T}$ cells. The FACS sorting (SONY Cell Sorter, SH800S) was used to isolate the $\mathrm{CD}^{+} \mathrm{YFP}^{+}$ Treg cells with purities $>99 \%$.

\section{Single-cell RNA sequencing}

$\mathrm{CD}^{+} \mathrm{YFP}^{+}$Treg cells were isolated from the peripheral lymph nodes of mice with indicated genotypes as above. To generate enough materials, the lymph nodes from 3 Foxp $3^{\text {cre/wt }}$ or 6 Foxp $3^{\text {cre/wt }}$;Rbx $1^{\text {fllfl }}$ mice at age of 10 weeks were pooled and subjected to single-cell RNA sequencing by Sinotech Genimics Co., Ltd. (Shanghai, China). The single-cell capture was achieved by random distribution of a single-cell suspension across $>200,000$ microwells through a limited dilution approach, and the $w t$ and Rbxl-deficient Treg cells were distributed on two individual microwell plates. Beads with oligonucleotide barcodes were added to saturation so that a bead was paired with a cell in a microwell. Cell-lysis buffer was added to facilitate poly-adenylated RNA molecules hybridized to the beads. Beads were collected into a single tube for reverse transcription. Upon cDNA synthesis, each cDNA molecule was 
tagged on the 5' end (that is, the 3' end of a mRNA transcript) with a molecular index and cell label to indicate its cell of origin. Whole transcriptome libraries were prepared using the BD Resolve system for single-cell whole-transcriptome amplification workflow (BD Genomics) to capture transcriptomic information of the sorted single cells. Briefly, the second strand cDNA was synthesized, followed by ligation of the adaptor for universal amplification. Eighteen cycles of PCR were used to amplify the adaptor-ligated cDNA products. Sequencing libraries were prepared using random priming PCR of the whole-transcriptome amplification products to enrich the 3' end of the transcripts linked with the cell labels and molecular indices. Sequencing libraries were quantified using a High Sensitivity DNA chip (Agilent) on a Bioanalyzer 2100 and the Qubit High Sensitivity DNA assay (Thermo Fisher Scientific). Approximate $1.5 \mathrm{pM}$ of the library for each sample was loaded onto a NextSeq 500 system and sequenced using High Output sequencing kits (75× 2bp) (Illumina). After discarding the cells with high mitochondrial gene, 10,048 and 9,736 cells were identified for $w t$ and Rbxl-deficient Treg cells, with an average number of reads of $23,321.27$ vs. 22202.55 , and $1,080.16$ vs. 870.28 of genes detected per cell, respectively.

\section{Transcriptome profiling}

$\mathrm{CD}^{+} \mathrm{YFP}^{+}$Treg cells were isolated from the peripheral lymph nodes and spleens of mice (8-12 weeks old) with indicated genotypes. To generate enough materials, the pooled tissues from 2-3 mice $\left(F_{o x p} 3^{\text {cre/wt }}\right)$, 8-11 (Foxp $\left.3^{\text {cre/wt }} ; R b x 1^{\text {flfft }}\right)$ or 3-4 $\left(\right.$ Foxp $3^{\text {cre/wt }} ; U$ Ube $\left.2 m^{f l f l}\right)$ were used. RNA samples were prepared with the miRNeasy Mini Kit (Qiagen, 21704), then reverse transcribed, amplified, labeled (Affymetrix GeneChip pico kit, 703308), and hybridized to Clariom S Arrays, mouse (Affymetrix, 902931), performed by Cnkingbio Biotech Co., Ltd. (Beijing, China). Microarray data sets were analyzed with Applied Biosystems Expression Console Software 1.4. Three independent experiments were performed.

\section{Proteomics profiling}

$\mathrm{CD}^{+} \mathrm{YFP}^{+}$Treg cells were isolated from the peripheral lymph nodes and spleens of mice ( $>8$ weeks of age) with indicated genotypes. To secure enough samples, tissues from 5-6 of Foxp $3^{\text {cre/wt }}$ or 21-24 of Foxp $3^{\text {cre/wt }}$;Rbx $1^{\text {flfl }}$ mice were pooled, washed with PBS buffer, and stored at $-80^{\circ} \mathrm{C}$. Three independent collected pooled samples were subjected to Mass-Spectrometry.

Cells were resuspended in $5 \mu 11 \%(\mathrm{w} / \mathrm{v})$ sodium deoxycholate, $10 \mathrm{mM}$ TCEP, $40 \mathrm{mM}$ 2-chloroacetamide (CAA), $100 \mathrm{mM}$ Tris ( $\mathrm{pH} 8.5$ ), and subsequently lysed by $5 \mathrm{~min}$ boiling at $95^{\circ} \mathrm{C}$ and sonication (Bioruptor, model UCD-200, Diagenode) for $15 \mathrm{~min}$. Cell debris were pelleted by centrifugation at 13,200r.p.m. for $5 \mathrm{~min}$ and the clarified lysate was transferred into a new vial. The lysate was diluted 1:10 for LysC-trypsin 
digestion $(0.4 \mu \mathrm{g}$ of each enzyme in double distilled water), and the digestion was performed overnight at $37^{\circ} \mathrm{C}$. The digest was acidified with $50 \mu 12 \%$ TFA and sodium deoxycholate was extracted using $50 \mu \mathrm{l}$ ethyl acetate and vigorous shaking. The organic phase was removed after centrifugation at 13,200r.p.m. for 5min. Finally, the peptides were desalted on C18 StageTips.

Samples were analyzed on Orbitrap Fusion Lumos mass spectrometers (Thermo Fisher Scientific, Rockford, IL, USA) coupled with an Easy-nLC 1000 nanoflow LC system (Thermo Fisher Scientific). Dried peptide samples were re-dissolved in Solvent A $(0.1 \%$ formic acid in water $)$ and loaded to a trap column $(100 \mu \mathrm{m} \times 2 \mathrm{~cm}$, home-made; particle size, $3 \mu \mathrm{m}$; pore size, $120 \AA \AA$; Dr. Maisch, Ammerbuch, Germany) with a max pressure of 280 bar using Solvent A, then separated on a home-made $150 \mu \mathrm{m} \times 30 \mathrm{~cm}$ silica microcolumn (particle size, $1.9 \mu \mathrm{m}$; pore size, $120 \AA$; Dr. Maisch, Ammerbuch, Germany) with a gradient of 5-35\% mobile phase B (acetonitrile and $0.1 \%$ formic acid) at a flow rate of $600 \mathrm{nl} / \mathrm{min}$ for $141 \mathrm{~min}$ then up to $95 \%$ in 1 minute and eluted for $9 \mathrm{~min}$ (the column temperature was maintained at $60^{\circ} \mathrm{C}$ ). The $\mathrm{MS}$ analysis were performed in data-dependent acquisition mode. Automatic gain control (AGC) targets were $5 \times 10^{5}$ ions with a max injection time of $50 \mathrm{~ms}$ for full scans and $5 \times 10^{3}$ with $35 \mathrm{~ms}$ for MS/MS scans. The most intense ions selected under top-speed mode were isolated in Quadrupole with a $1.6 \mathrm{~m} / \mathrm{z}$ window and fragmented by higher energy collisional dissociation (HCD) with normalized collision energy of $32 \%$ and dynamic exclusion time was set as $25 \mathrm{~s}$. Data were acquired using the Xcalibur software (Thermo Fisher Scientific).

DAVID was used for pathway enrichment analysis of proteins with change fold $>2 / 3$ or $<0.5 / 0.33$. The enriched pathways and their contained proteins with 2 -fold change were shown in heat map in which each quantitative value was normalized based its maximum value.

\section{Statistical analysis}

The $p$ values were calculated by Mann-Whitney test, two-tailed unpaired Student's t-test, one-way ANOVA or two-way ANOVA as indicated using GraphPad Prism, unless otherwise noted. Statistical analysis of mouse survival and respective $p$ values were determined using the log-rank test. $p<0.05$ was considered as significant. All error bars represent the s.e.m. from three independent experiments.

\section{Data availability}

The scRNA-seq and microarray data that support the findings of this study will be deposited in the Gene Expression Omnibus. 


\section{References}

1. Kamura, T. et al. Rbx1, a component of the VHL tumor suppressor complex and SCF ubiquitin ligase. Science 284, 657-661 (1999)

2. Ohta, T. Michel, J. J., Schottelius, A. J., Xiong, Y. ROC1, a homolog of APC11, represents a family of cullin partners with an associated ubiquitin ligase activity. Mol Cell 3, 535-541 (1999)

3. Seol, J. H. et al. Cdc53/cullin and the essential Hrt1 RING-H2 subunit of SCF define a ubiquitin ligase module that activates the E2 enzyme Cdc34. Genes Dev 13, 1614-1626 (1999)

4. Tan, P. et al. Recruitment of a ROC1-CUL1 ubiquitin ligase by Skp1 and HOS to catalyze the ubiquitination of IкB $\alpha$. Mol Cell 3, 527-533 (1999)

5. Huang, D. T. et al. E2-RING Expansion of the NEDD8 Cascade Confers Specificity to Cullin Modification. Mol Cell 33, 483-495 (2009)

6. Baek, K. et al. NEDD8 nucleates a multivalent cullin-RING-UBE2D ubiquitin ligation assembly. Nature 578: 461-466 (2020)

7. Hori, S., Nomura, T, \& Sakaguchi, S. Control of regulatory T cell development by the transcription factor Foxp3. Science 299, 1057-1061 (2003)

8. Fontenot, J. D., Gavin, M. A., \& Rudensky, A. Y. Foxp3 programs the development and function of $\mathrm{CD} 4{ }^{+} \mathrm{CD} 25^{+}$regulatory T cells. Nat Immunol 4, 330-336 (2003)

9. Khattri, R. et al. An essential role for Scurfin in $\mathrm{CD} 4^{+} \mathrm{CD} 25^{+} \mathrm{T}$ regulatory cells. Nat Immunol 4, 337-342 (2003)

10. Kim, J. M., Rasmussen, J. P., \& Rudensky, A.Y. Regulatory T cells prevent catastrophic autoimmunity throughout the life span of mice. Nat Immunol 8, 191-197 (2007)

11. Soucy, T. A. et al. An inhibitor of NEDD8-activating enzyme as a new approach to treat cancer. Nature 458, 732-736 (2009)

12. Liu, J. et al. Targeting SCF E3 ligases for cancer therapies. Adv Exp Med Biol 1217, 123-146 (2020)

13. Tan, M., Davis, S. W., Saunders, T. L., Zhu, Y., \& Sun, Y. RBX1/ROC1 disruption results in early embryonic lethality due to proliferation failure, partially rescued by simultaneous loss of p27. Proc Natl Acad Sci U S A 106, 6203-6208 (2009)

14. Tan, M. et al. SAG/RBX2/ROC2 E3 ubiquitin ligase is essential for vascular and neural development by targeting NF1 for degradation. Dev Cell 21, 1062-1076 (2011)

15. Mathewson N. D. et al. SAG/Rbx2-Dependent neddylation regulates T-cell responses. Am J Pathol 186, 2679-2691 (2016)

16. Xiong $X$. et al. SAG/RBX2 E3 ubiquitin ligase differentially regulates inflammatory responses of myeloid cell subsets. Front Immunol 9, 2282 (2018)

17. Rubtsov, Y. P. et al. Regulatory T cell-derived interleukin-10 limits inflammation at environmental interfaces. Immunity 28, 546-558 (2008) 
18. Wu, D. et al. Lkb1 maintains Treg cell lineage identity. Nat Commun 8, 15876 (2017)

19. Gavin M. A. et al. Foxp3-dependent programme of regulatory T-cell differentiation. Nature 445, 771-775 (2007)

20. Taylor, P. A. et al. L-Selectin(hi) but not the L-selectin(lo) CD4 ${ }^{+} 25^{+}$T-regulatory cells are potent inhibitors of GVHD and BM graft rejection. Blood 104, 3804-3812 (2004)

21. Read, S., Malmstrom, V., Powrie, F. Cytotoxic T lymphocyte-associated antigen 4 plays an essential role in the function of CD25(+)CD4 $(+)$ regulatory cells that control intestinal inflammation. $J$ Exp Med 192, 295-302 (2000)

22. Herman, A. E., Freeman, G. J., Mathis, D., Benoist, C. CD4 ${ }^{+} \mathrm{CD} 25^{+}$T regulatory cells dependent on ICOS promote regulation of effector cells in the prediabetic lesion. J Exp Med 199, 1479-1489 (2004)

23. Firan M., Dhillon S., Estess P., Siegelman M. H. Suppressor activity and potency among regulatory $\mathrm{T}$ cells is discriminated by functionally active CD44. Blood 107, 619-627 (2006)

24. Ricardo, J. M. et al. Single-cell transcriptomics of regulatory T cells reveals trajectories of tissue adaptation. Immunity 50, 493-504 (2019)

25. Li M. O., Rudensky A. Y., T cell receptor signalling in the control of regulatory $\mathrm{T}$ cell differentiation and function. Nat Rev Immunol 16, 220-233 (2016)

26. Joetham, A., et al. Naturally occurring lung CD4(+)CD25(+) T cell regulation of airway allergic responses depends on IL-10 induction of TGF-beta. J Immunol 178, 1433-1442 (2007)

27. Zacchigna, S., et al. Paracrine effect of regulatory T cells promotes cardiomyocyte proliferation during pregnancy and after myocardial infarction. Nat commun 26, 2432 (2018)

28. Chinen, T., et al. An essential role for the IL-2 receptor in Treg cell function. Nat Immunol 17, 1322-1333 (2016)

29. Deaglio, S., et al. Adenosine generation catalyzed by CD39 and CD73 expressed on regulatory T cells mediate immune suppression. J Exp Med 204, 1257-1265 (2007) 30. Borsellino, G., et al. Expression of ectonucleotidase CD39 by Foxp3 ${ }^{+}$Treg cells: hydrolysis of extracellular ATP and immune suppression. Blood 110, 1225-1232 (2007)

31. Delgoffe, G. M., et al. Stability and function of regulatory T cells is maintained by a neuropilin-1-semaphorin-4a axis. Nature 501, 252-256 (2013)

32. Huang, C. T., et al. Role of LAG-3 in regulatory T cells. Immunity 21, 503-513 (2004)

33. Venet, F., et al. Human CD4+CD25+ regulatory $\mathrm{T}$ lymphocytes inhibit lipopolysaccharide-induced monocyte survival through a Fas/Fas ligand-dependent mechanism. J Immunol 177, 6540-6547 (2006) 
34. Garin, M. I. et al. Galectin-1: A key effector of regulation mediated by CD4+CD25+ T cells. Blood 109, 2058-2065 (2007)

35. Wang D. et al. A deep proteome and transcriptome abundance atlas of 29 healthy human tissues. Mol Syst Biol 15, e8503 (2019)

36. Zhang C., et al. Cullin3-KLHL25 Ubiquitin Ligase Targets ACLY for Degradation to Inhibit Lipid Synthesis and Tumor Progression. Genes Dev 30, 1956-1970 (2016)

37. Nakayama K., et al. Impaired Degradation of Inhibitory Subunit of NF-kappa B (I Kappa B) and Beta-Catenin as a Result of Targeted Disruption of the beta-TrCP1 Gene. Proc Natl Acad Sci U S A 100, $8752-8757$ (2003)

38. Zhao Y., Sun Y. Cullin-RING Ligases as Attractive Anti-cancer Targets. Curr Pharm Des 19, 3215-3225 (2013)

39. Zhou L., Zhang W., Sun Y., Jia L. Protein neddylation and its alterations in human cancers for targeted therapy. Cell Signal 44, 92-102 (2018)

40. Huang $H$. et al. Skp2 inhibits FOXO1 in tumor suppression through ubiquitin-mediated degradation. Proc Natl Acad Sci U S A 102, 1649-1654 (2005)

41. Chang M., Lee A. J., Fitzpatrick L., Zhang M., Sun S. C. NF-kappa B1 p105 regulates $\mathrm{T}$ cell homeostasis and prevents chronic inflammation. J Immunol 182, 3131-3138 (2009)

42. Tong, J. et al. $\mathrm{m}^{6} \mathrm{~A}$ mRNA methylation sustains Treg suppressive functions. Cell Res 28, 253-256 (2018)

43. Zhang M. et al. The roles of Egr-2 in autoimmune diseases. Inflammation 38, 972-977 (2015)

44. Iellem A. et al. Unique chemotactic response profile and specific expression of chemokine receptors CCR 4 and CCR 8 by CD4(+)CD25(+) regulatory T cells. $J$ Exp Med 194, 847-853 (2001)

45. Joller N. et al. Treg cells expressing the coinhibitory molecule TIGIT selectively inhibit proinflammatory Th1 and Th17 cell responses. Immunity 40, 569-581 (2014)

46. $\mathrm{Li} \mathrm{H}$. et al. Inactivation of SAG/RBX2 E3 ubiquitin ligase suppresses $\mathrm{Kras}^{\mathrm{G} 12 \mathrm{D}}$-driven lung tumorigenesis. J Clin Invest 124, 835-46 (2014) 

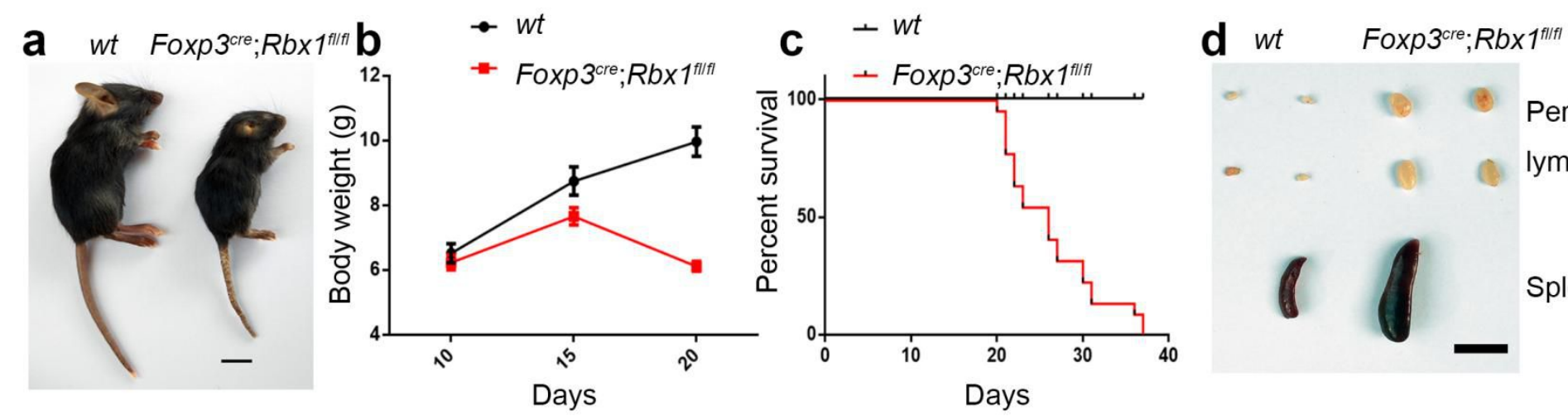

e $\quad w t$
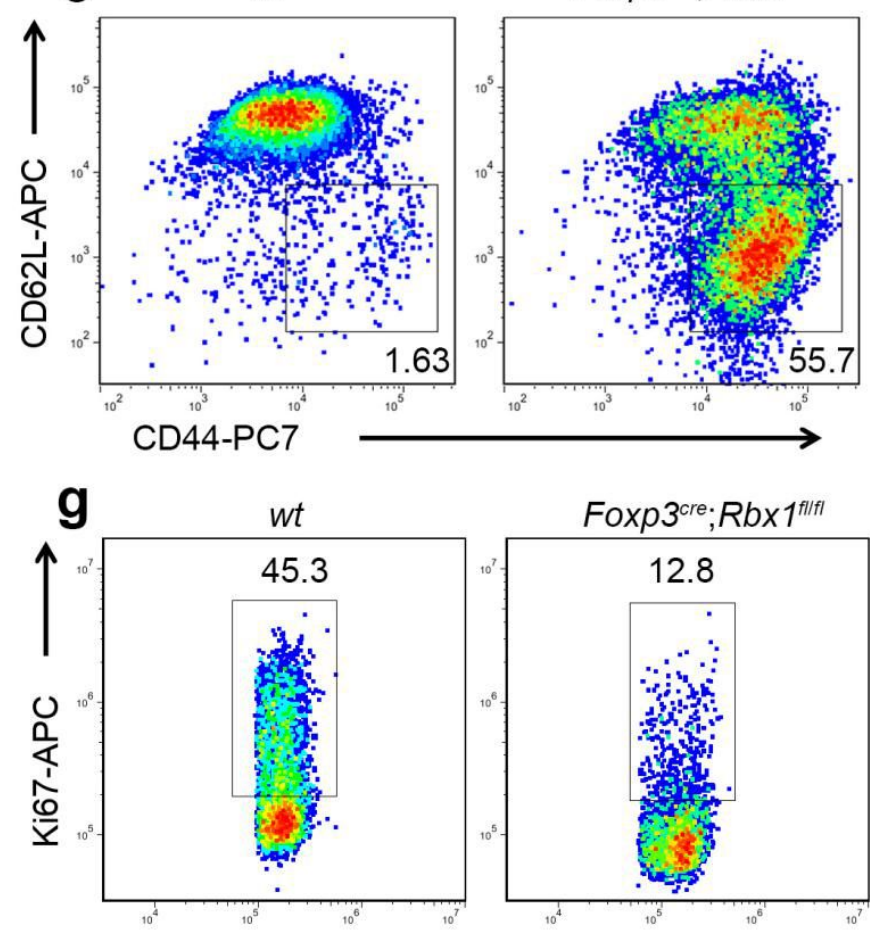

Foxp3-PE

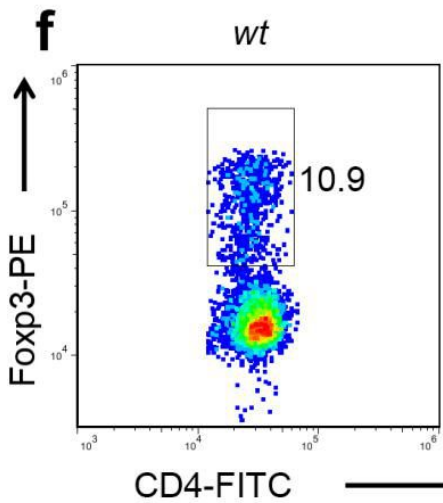

h

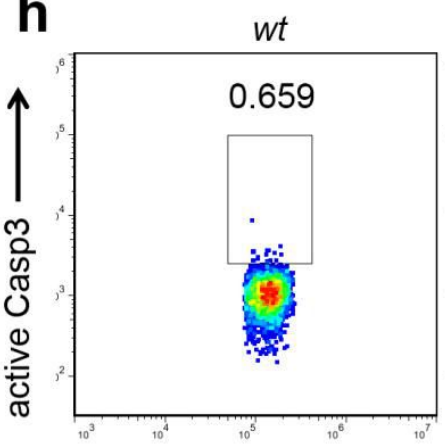

Foxp3-A488
Peripheral lymph nodes

Spleen

i
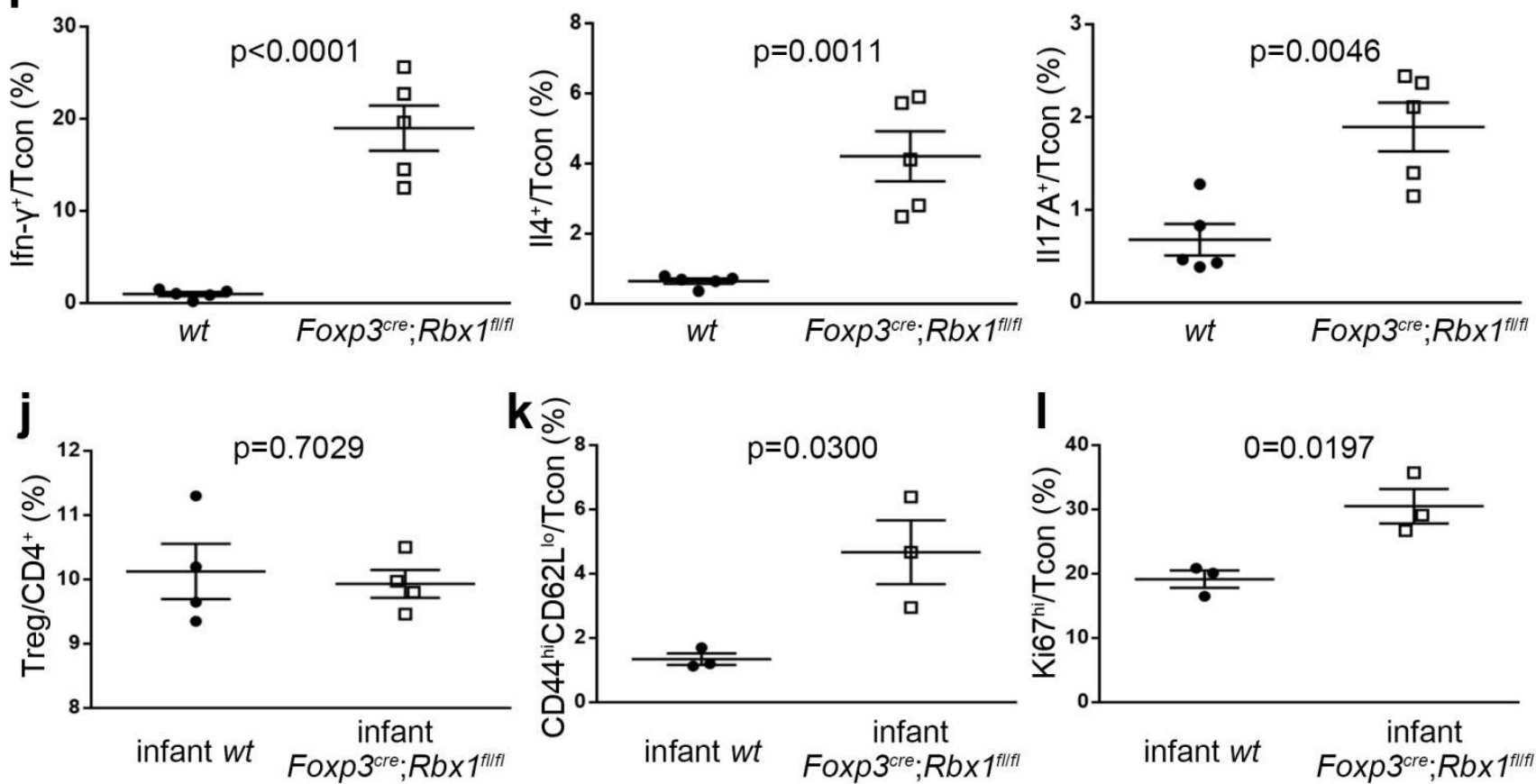
Figure 1. $R b x \boldsymbol{l}$ deletion in Treg cells leads to an early-onset fatal inflammatory disorder

a. Representative images of $w t$ and $F o x p 3^{\text {cre }} ; R b x 1^{f l f l}$ mice $(\mathrm{p} 20$, scale bar $=1 \mathrm{~cm})$.

b. Gross body weight of $w t$ and Foxp $3^{\text {cre }} ; R b x l^{f l f l}$ mice $(n=10)$.

c. Survival curve of $w t$ and Foxp $3^{\text {cre }} ; R b x 1^{f l f l}$ mice $(n=21 ; p<0.0001)$.

d. Representative images of the peripheral lymph nodes and spleen from $w t$ and Foxp $3^{\text {cre }} ; R b x l^{f l f l}$ mice (p22, scale bar $\left.=1 \mathrm{~cm}\right)$.

e. Expression of CD44 and CD62L in Tcon cells from peripheral lymph nodes of $w t$ and Foxp3 $3^{\text {cre }} ; R b x 1^{f l f l}$ mice (p21).

f. The proportion of Treg cells among $\mathrm{CD}^{+}-\mathrm{T}$ cells from peripheral lymph nodes of $w t$ and Foxp $3^{\text {cre }} ; R b x l^{f l l f l}$ mice (p23).

g. Expression of Ki67 in Treg cells from peripheral lymph nodes of $w t$ and Foxp3 $3^{\text {cre }} ;$ Rbx $1^{\text {fllfl }}$ mice (p19-23, $\left.n=5\right)$.

h. Active Casp3 in Treg cells from peripheral lymph nodes of $w t$ and Foxp ${ }^{\text {cre }} ; R b x I^{f l f l}$ mice (p19-23, $n=4)$.

i. The levels of Ifn- $\gamma$, Il-4 and Il-17 in Tcon cells from peripheral lymph nodes in $w t$ and Foxp3 ${ }^{\text {cre }} ;$ Rbx $I^{\text {fllfl }}$ mice (p19-23, $\left.n=5\right)$.

j-1. Impaired suppressive function of Rbx1-deficient Treg cells. j,Treg/CD4 ${ }^{+}$ratios (p8); k, $\mathrm{CD} 44^{\mathrm{hi}} \mathrm{CD} 62 \mathrm{~L}^{\mathrm{lo}} / \mathrm{Tcon}$ ratios $(\mathrm{p} 8) ; 1, \mathrm{Ki} 67^{\mathrm{hi}} / \mathrm{Tcon}$ ratios $(\mathrm{p} 8)$ from peripheral lymph nodes of $w t$ and Foxp $3^{\text {cre }} ; R b x 1^{f l f l}$ infant mice at p8. $(n=3-4)$. 
bioRxiv preprint doi: https://doi.org/10.1101/2020.07.31.230532; this version posted July 31, 2020. The copyright holder for this preprint (which was not certified by peer review) is the author/funder. All rights reserved. No reuse allowed without permission.

a
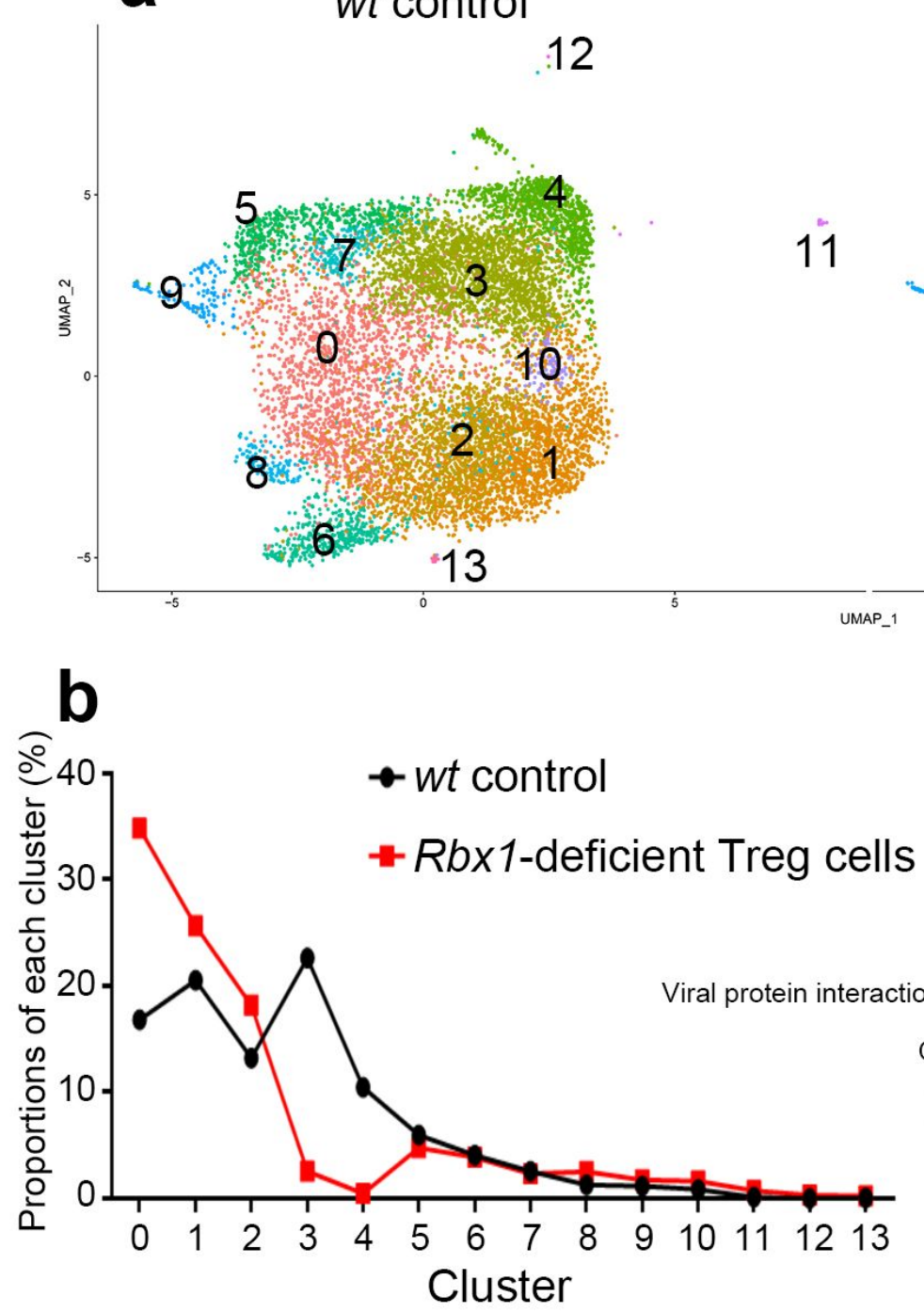

C

0

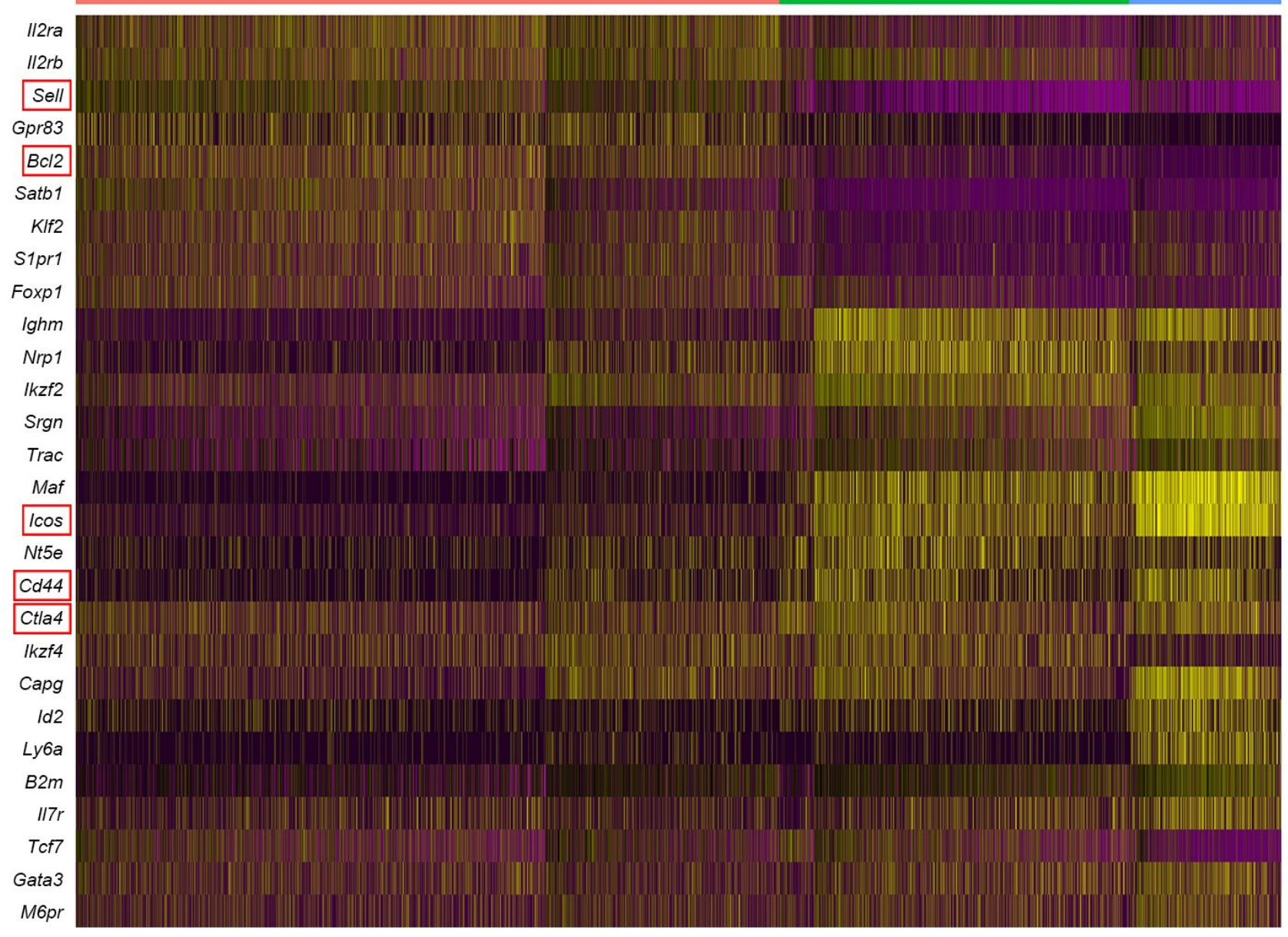

Rbx1-deficient Treg cells

12

11

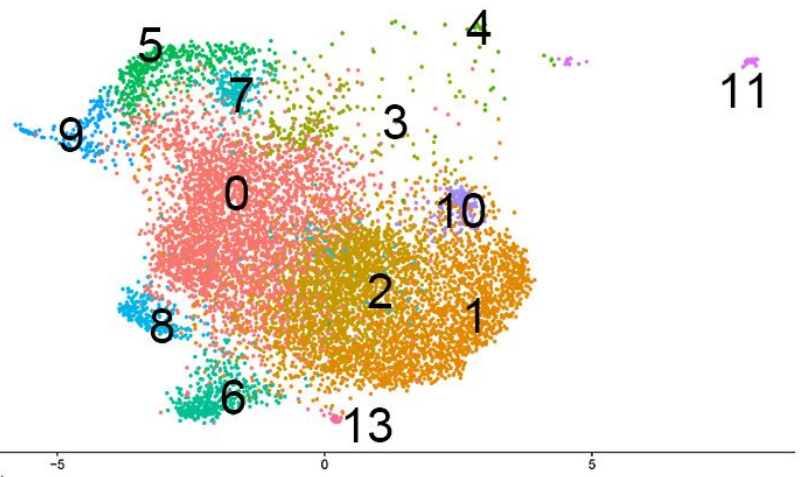




\section{Figure 2. Single-cell transcriptomics of $w t$ and $R b x 1$-deficient Treg cells}

a. Two-dimensional UMAP visualization of single cell transcriptomics data in $\mathrm{CD}^{+} \mathrm{YFP}^{+}$ Treg cells from peripheral lymph nodes of Foxp $3^{\text {cre/wt }}$ and Foxp $3^{\text {cre/wt }} ; R b x 1^{f l l f l}$ mice $(10$ weeks old).

b. Proportions of each cluster in $\mathrm{CD}^{+} \mathrm{YFP}^{+}$Treg cells from peripheral lymph nodes of Foxp $3^{\text {crelWT }}$ and Foxp $3^{\text {crelwt }} ;$ Rbx $l^{\text {flfl }}$ mice (10 weeks old).

c. Heat map of selected marker genes in clusters 0, 3 and 4. Single cells were represented by vertical lines and different colors reflected the relative abundance of indicated genes.

d. Selected pathways of clusters 0,3 and 4 in the KEGG analysis. 


\section{Figure 3. Rbx1-dependent transcriptome and proteome in Treg cells}

a. Differentially expressed genes related to Treg function in $\mathrm{CD}^{+} \mathrm{YFP}^{+}$Treg cells from Foxp $3^{\text {cre/wt }}$ and Foxp $3^{\text {cre/wt }}$;Rbx $1^{f l f l}$ mice (8-10 weeks old), determined by transcriptional profiling.

b. Comparison of expression of genes associated with indicated pathways in $\mathrm{CD}^{+} \mathrm{YFP}^{+}$Treg cells derived from Foxp $3^{\text {cre/wt }}$ and Foxp $3^{\text {cre/wt }} ;$ Rbx $1^{\text {flflf }}$ mice (8-10 weeks old).

c. Comparison of the levels of proteins vs. mRNAs in genes associated with indicated pathways in $\mathrm{CD}^{+} \mathrm{YFP}^{+}$Treg cells from Foxp $3^{\text {cre/wt }}$ and Foxp $3^{\text {crelwt }} ; R$ Rbx $1^{\text {flfl } l}$ mice $(8-10$ weeks old). The mRNAs with greater than 1.3 -fold changes, which are consistent with protein level changes, were marked by the arrows.

d. Comparison of the levels of proteins vs. mRNAs in indicated genes in $\mathrm{CD}^{+} \mathrm{YFP}^{+}$Treg cells from Foxp $3^{\text {cre/wt }}$ and Foxp $3^{\text {crelwt }} ; R b x 1^{\text {fllfl }}$ mice (8-10 weeks old). Those with increased levels of protein but not the mRNA were considered as candidates of the direct degradation substrates of Rbx1 E3 ligase in Treg cells. 


\section{Figure 4. Ube2m-dependent and -independent mechanisms of Rbx1 in Treg cells}

a. Representative images of $w t$ and Foxp $3^{\text {cre }} ;$ Ube $2 m^{f l f l}$ mice $(16$ weeks old, scale bar $=$ $1 \mathrm{~cm})$.

b. Gross body weight of $w t$ and Foxp $3^{\text {cre }} ; U b e 2 m^{\text {fllfl }}$ mice $(n=20)$.

c. Survival curve of $w t$ and Foxp $3^{\text {cre }} ; U b e 2 m^{f l f l}$ mice $(n=12 ; p<0.0001)$.

e. Representative images of the peripheral lymph nodes and spleen from $w t$ and Foxp $3^{\text {cre }}$ Ube $2 m^{\text {fllfl }}$ mice (16 weeks old, scale bar $=1 \mathrm{~cm}$ ).

e. Expression of CD44 and CD62L in Tcon cells from peripheral lymph nodes of $w t$ and Foxp $3^{\text {cre }} ;$ Ube $2 m^{\text {fllfl }}$ mice (16 weeks old).

f. The levels of Ifn- $\gamma$, Il-4 and Il-17 in Tcon cells from peripheral lymph nodes of $w t$ and Foxp $3^{\text {cre }} ; U b e 2 m^{\text {fllfl }}$ mice (16 weeks old, $n=4$ )

g. Unbiased cluster analysis of the transcriptional programs revealed 4 categories of genes differentially expression in Rbxl-and Ube $2 m$-deficient Treg cells, as compared to the $w t$ control Treg cells. 